\title{
MicroRNA-124 suppresses cell proliferation and invasion of triple negative breast cancer cells by targeting STAT3
}

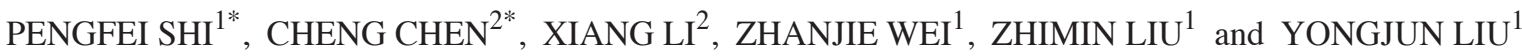 \\ ${ }^{1}$ Department of Thyroid and Breast Surgery, The Central Hospital of Wuhan; ${ }^{2}$ Department of General Surgery, Union Hospital, \\ Tongji Medical College, Huazhong University of Science and Technology, Wuhan, Hubei 430000, P.R. China
}

Received July 19, 2018; Accepted March 6, 2019

DOI: $10.3892 / \mathrm{mmr} .2019 .10044$

\begin{abstract}
MicroRNAs (miRNAs) are pivotal regulators of the progression of carcinogenesis and negatively regulate the expression of tumour-associated genes. Downregulation of miR-124 expression has been demonstrated in various human cancer tissues, wherein miR-124 serves as a tumour suppressor by targeting oncogenes. However, its function and underlying mechanism of action remain unclear in breast cancer. In the present study, the tissue-specific expression of miR-124 was detected in 10 paired triple-negative breast cancer and normal tissues, and its inhibitory effects on cell growth and invasion were evaluated in vitro and in vivo. Bioinformatics analysis identified signal transducer and activator of transcription 3 (STAT3), a well-known oncogene in breast cancer, as the potential target. Upregulation of miR-124 expression decreased STAT3 mRNA and protein levels in breast cancer cells and the relative luciferase activity. Rescue experiments revealed that the transfection of a STAT3 expression plasmid reversed the inhibitory effect of miR-124 on the proliferation and invasion of MDA-MB-468 cells. These data demonstrate that miR-124 serves vital roles in the suppression of triple-negative breast cancer via inhibition of cell proliferation and invasion through STAT3. These results highlight the potential role of miR-124 as a diagnostic or therapeutic target in patients with breast cancer.
\end{abstract}

\section{Introduction}

Breast cancer is the most common cancer in females worldwide (1). Understanding of the mechanism underlying

Correspondence to: Dr Yongjun Liu, Department of Thyroid and Breast Surgery, The Central Hospital of Wuhan, Tongji Medical College, Huazhong University of Science and Technology, 26 Shengli Road, Jiangan, Wuhan, Hubei 430000, P.R. China E-mail: chenc5230@126.com

\section{${ }^{*}$ Contributed equally}

Key words: breast cancer, microRNA-124, signal transducer and activator of transcription 3 , proliferation, invasion tumour progression and the identification of novel therapeutic methods are required to improve the prognosis of patients with breast cancer. In previous decades, studies have primarily focused on alterations in mRNA expression in cancer cells; however, recent studies have suggested the important roles for non-coding RNAs in the regulation of cell proliferation, apoptosis, chemo-resistance and migration in cancer (2-5).

MicroRNAs (miRNAs/miRs) are non-coding endogenous RNAs measuring 22 nucleotides long. miRNAs bind to the partially complementary sequences in the 3'-untranslated region (3'-UTR) of the target mRNAs and stimulate mRNA degradation or translation inhibition $(6,7)$. Aberrant expression of multiple miRNAs has been identified in breast cancer, and their expression levels were associated with the extent of invasion or proliferation of cancer cells (8-10). Subsequent exploration into the role of the tumour-associated miRNAs in breast cancer may allow identification of novel therapeutic targets to improve clinical outcomes. miR-124 is significantly downregulated in various types of cancer, including colorectal cancer (CRC) (11), non-small cell lung cancer (12), and nasopharyngeal carcinoma (NPC) (13). It serves as a tumour suppressor gene and affects apoptosis, proliferation and invasion of cells. The expression of miR-124 was downregulated in CRC tissues and its upregulated expression may suppress tumour progression and decrease the drug sensitivity of CRC cells through the negative regulation of the expression of DNA methyltransferase (DNMT) 3 beta and DNMT1 (11). Decreased expression of miR-124 was also observed in NPC tissues, wherein it contributed to the initiation and development of NPC by targeting forkhead box Q1, which is involved in tumour proliferation and migration (13). Downregulation of miR-124 expression has also been demonstrated in human breast cancer tissues $(14,15)$. miR-124 serves a vital role in the modulation of proliferation and invasion of breast cancer cells through the downregulation of the oncogene Cbl proto-oncogene (16). In addition, miR-124 mediated the oncogenic effects of metastasis-associated lung adenocarcinoma transcript 1 in breast cancer by directly interacting with the cyclin-dependent kinase 4/E2F transcription factor 1 signalling pathway (17). Therefore, miR-124 is an attractive candidate as a therapeutic target in breast cancer. However, the effects and underlying mechanisms of this miRNA in breast cancer are incompletely characterized. 
The present study aimed to clarify the functions of miR-124 in breast cancer cells and to examine its effects on the viability, proliferation and invasion of breast cancer cell lines. Furthermore, these data were validated by performing in vivo studies. Bioinformatics software was used to predict the potential targets of miR-124 in breast cancer cells. According to the results, signal transducer and activator of transcription 3 (STAT3) was identified as the target. STAT3 is a key cytoplasmic transcription factor involved in cell proliferation and invasion and serves as an oncogenic gene in breast cancer (18-20). STAT3 may bind to the promoter of the tumour protein p53 (p53) gene and inhibit its expression, resulting in the inhibition of $\mathrm{p} 53$-mediated apoptosis of cancer cells. In addition, miR-17-5p may suppress the chemotherapy-induced apoptosis of breast cancer cells through the deactivation of STAT3 (18). Liu et al (20) demonstrated that STAT3 may enhance the transcription and expression of mitogen-activated protein kinase kinase 5 to promote epithelial-mesenchymal transition (EMT) in breast cancer cells. Previous data have suggested that STAT3 is upregulated in breast cancer tissues, and the small-interfering RNA (siRNA) and miRNA-mediated inhibition of STAT3 expression resulted in suppression of the invasion of breast cancer cells $(19,21,22)$. The present study evaluated if miR-124 exerted its anti-proliferation and anti-invasion effects by targeting STAT3. The results indicated that miR-124 targeted STAT3 to decrease the growth rate and invasiveness of breast cancer cells in vitro and in vivo.

\section{Patients and methods}

Human tissues samples. Human breast tissue samples were obtained at the Central Hospital of Wuhan (Wuhan, China) from September 2014 to November 2015. A total of 10 patients (42-57 years old) with triple negative breast cancer were included in the study. Cancer tissues and matched normal breast tissues were collected. All patients from whom samples were collected were treatment-naïve prior to surgical resection. The study protocol followed the Declaration of Helsinki, and all patients provided written informed consent. All protocols and procedures were approved by the Ethics Committee of the Central Hospital of Wuhan.

Cell culture. The human breast cancer cell lines MDA-MB-468 and MDA-MB-231 were obtained from the American Type Culture Collection (Manassas, VA, USA). MDA-MB-468 cells were routinely cultured at $37^{\circ} \mathrm{C}$ in a $5 \% \mathrm{CO}_{2}$ environment with $\mathrm{L} 15,10 \%$ foetal bovine serum (FBS), $100 \mathrm{U} / \mathrm{ml}$ penicillin and streptomycin (all Gibco; Thermo Fisher Scientific, Inc., Waltham, MA, USA). MDA-MB-231 cells were cultured without $\mathrm{CO}_{2}$ in complete L15 medium (Gibco; Thermo Fisher Scientific, Inc), according to the protocol of the manufacturer.

Transfection. miRNA-124 mimics (miR-124), miRNA-124 inhibitor (inh-124), STAT3 overexpression plasmid [pcDNA3.0-STAT3 (plasmid-STAT3)] and corresponding negative controls [miR-NC, inh-NC and pcDNA3.0-empty vector (plasmid-NC)] were designed and purchased from Guangzhou RiboBio Co., Ltd. (Guangzhou, China). The sequences of miR-124, miR-NC, inh-124 and inh-NC were 5'-CCGUAAGUGGCGCACGGAAU-3', 5-UUCUCCGAA CGUGUCACGUTT-3', 5'-GGCAUUCACCGCGUGCCU
UA-3' and 5'-CAGUACUUUUGUGUAGUACAA-3', respectively. The sense and antisense of si-STAT3 were 5'-CAU CUGCCUAGAUCGGCUA-3' and 5'-UAGCCGAUCUAG GCAGAUG-3'; the sequences for negative control (si-NC) were 5'-UUCUCCGAACGUGUCACGUTT-3' and 5'-ACG UGACACGUUCGGAGAATT-3'. Based on these sequences, lentiviral vectors (pHelper 2.0) with siRNA targeting human STAT3 (LV-si-STAT3), LV-miR-124, and corresponding controls (LV-si-NC/LV-miR-NC) were purchased from Shanghai GeneChem Co., Ltd. (Shanghai, China). A total of $5 \times 10^{4}$ cells were seeded in 12-well plates and incubated overnight at $37^{\circ} \mathrm{C}$ in Opti-MEM medium (Gibco; Thermo Fisher Scientific, Inc.), followed by RNA/DNA transfection using Lipofectamine ${ }^{\circledR} 2000$ (Invitrogen; Thermo Fisher Scientific, Inc.). The final concentrations of miR-124/miR-NC and inh-124/inh-NC were 50 and $100 \mathrm{nM}$, respectively. Cells were transfected with plasmid-STAT3 at a concentration of $1.6 \mu \mathrm{g} /$ well in a 12 -well plate. For lentiviral transfection, the concentration was set as $1 \times 10^{8}$ transducing units $/ \mathrm{ml}$ in 6-well plates. Western blot analysis and reverse transcription quantitative polymerase chain reaction (RT-qPCR) assays were performed, as described subsequently, to evaluate the transfection efficiency $48 \mathrm{~h}$ following transfection. For lentiviral infection, the cells were cultured at $37^{\circ} \mathrm{C}$ in Enhanced Infection Solution and Polybrene (both Shanghai GeneChem Co., Ltd.) for $5 \mathrm{~h}$, and then this mixture was replaced with complete medium.

Total RNA isolation and RT-qPCR. According to the manufacturer's protocol, TRIzol ${ }^{\circledR}$ (Takara Bio, Inc., Otsu, Japan) was used to extract total RNA from breast cancer cells $48 \mathrm{~h}$ after treatment. mRNAs and miRNAs were reverse transcribed according to the suppliers' protocols using PrimeScript ${ }^{\circledR}$ RT Master Mix Perfect Real Time and One Step PrimeScript ${ }^{\circledR}$ microRNA cDNA Synthesis kits (both Takara Bio, Inc.), respectively. SYBR Premix Ex Taq II (Takara Bio, Inc.) was used for qPCR analysis. The qPCR conditions were as follows: $95^{\circ} \mathrm{C}$ for $30 \mathrm{sec}$, followed by 40 cycles of $95^{\circ} \mathrm{C}$ for $5 \mathrm{sec}$ and $60^{\circ} \mathrm{C}$ for $30 \mathrm{sec}$. The $2^{-\Delta \Delta \mathrm{Cq}}$ method was used to quantify expression (23). The expression levels of mRNAs and miRNAs were normalized to $\beta$-actin and U6, respectively, and all reactions were performed in triplicate. The primers for the RT-qPCR were: miR-124 forward, 5'-TAAGGCACGCGG TGAATGCC-3' and reverse, 5'-GATTGAATCGAGCAC CAGTTA'-3; U6 forward, 5'-GCTTCGGCAGCACATATA CTAAAAT-3' and reverse, 5'-CGCTTCACGAATTTGCGT GTCAT-3'; STAT3 forward, 5'-TGTGCGTATGGGAACACC TA-3' and reverse, 5'-AGAAGGTCGTCTCCCCCTTA-3'; $\beta$-actin forward, 5'-CTTTCTACAATGAGCTGCGTG-3' and reverse, 5'-TCATGAGGTAGTCTGTCAGG-3'.

Western blot analysis. Transfected cells were washed with PBS three times prior to protein collection. Protein lysis buffer was obtained from Cell Signaling Technology, Inc. (Danvers, MA, USA), and a bicinchoninic acid assay was performed to measure the concentration of protein. Protein samples $(30 \mu \mathrm{g} /$ lane) were separated via 10\% SDS-PAGE and transferred to polyvinylidene difluoride membranes. Then, the membranes were blocked in 5\% skimmed milk dissolved in TBS- $0.1 \%$ Tween-20 for $2 \mathrm{~h}$ at room temperature and 
further incubated at $4^{\circ} \mathrm{C}$ overnight with the primary antibodies rabbit anti-STAT3 (1:1,000; cat. no. 12640) and mouse anti- $\beta$-actin $(1: 1,000$; cat. no. 3700 ; both Cell Signaling Technology, Inc.). Following washing, the membranes were incubated with horseradish peroxidase-conjugated anti-rabbit and anti-mouse secondary antibodies (1:3,000; cat. nos. 7074 and 7076; Cell Signaling Technology, Inc.) at room temperature for $2 \mathrm{~h}$. Bands were visualized and quantified using enhanced chemiluminescent substrate reagents (Thermo Fisher Scientific, Inc.) and Image Lab version 4.1 software (Bio-Rad Laboratories, Inc., Hercules, CA, USA), respectively.

MTT assay. An MTT assay was conducted to measure the viability of cancer cells. Cells were seeded at a density of $3 \times 10^{3}$ cells per well for a 12 -well plate. Every group contained at least 6 wells. Cells were then cultured overnight and transfected as aforementioned with mimics (50 nM)/inhibitor $(100 \mathrm{nM})$ and corresponding negative controls, respectively. Cells were cultured for 2 days prior to the MTT assay to determine the effects of treatment on cell viability. Cells were incubated with MTT $(5 \mathrm{mg} / \mathrm{ml})$ at $37^{\circ} \mathrm{C}$ for $4 \mathrm{~h}$, and then $150 \mu \mathrm{l}$ dimethyl sulfoxide was added. The crystals were dissolved, and the absorbance was measured using a spectrophotometer at $570 \mathrm{~nm}$. For measuring cell viability following transfection with lentiviruses, stably-transfected cells were plated in 5 plates and MTT assays were conducted to detect the relative absorbance every day; growth curves were calculated following 5 days of observation. All procedures were repeated 3 times.

Cell invasion assay. Invasion assays in the present study were performed in triplicate using Transwell chambers with $8 \mu \mathrm{m}$ pore size (Costar; Corning Incorporated, Corning, NY, USA), which were coated with 1:10 diluted Matrigel (BD Biosciences, San Jose, CA, USA) for $4 \mathrm{~h}$ at $37^{\circ} \mathrm{C}$. A total of $\sim 5 \times 10^{4}$ cells were plated in $200 \mu \mathrm{l}$ of serum-free L15 medium in the upper chamber. Then, $700 \mu 1$ L15 medium containing $30 \%$ FBS was placed in the lower chambers. Following incubation for $48 \mathrm{~h}$ at $37^{\circ} \mathrm{C}$, the cells on the top were removed, and cells on the lower surface were stained at $37^{\circ} \mathrm{C}$ for $15 \mathrm{~min}$ with $0.1 \%$ crystal violet solution and counted in 10 microscopic fields using a light microscope (magnification, $\mathrm{x} 400$ ).

Dual-luciferase reporter assay. Putative binding sequences of miR-124 and STAT3 mRNA were predicted using PITA version 6 bioinformatics (24). Wild type (WT) or mutant (MUT) dual luciferase reporter plasmids (pmiR-RB-Report ${ }^{\mathrm{TM}}$ ) were designed to contain the original binding sequence of STAT3 mRNA or a non-functional MUT sequence (Guangzhou RiboBio Co., Ltd.). A total of $5 \times 10^{3}$ cells/well were plated in a 96-well plate and then co-transfected with luciferase reporter plasmids and miR-124 or miR-NC using Lipofectamine 2000. Then, 2 days post-transfection, a Dual-Luciferase Reporter Assay System (Promega Corporation, Madison, WI, USA) was applied to quantify the luciferase activity. For data analysis, firefly luciferase activity was normalized to the corresponding Renilla luciferase activity. All experiments were performed 3 times with 5 duplicates.
Nude mouse models. A total of 20 male BALB/c nude mice (3 weeks old) were purchased from Beijing HFK Bioscience Co., Ltd. (Beijing, China) and housed in a room with controlled temperature $\left(26-28^{\circ} \mathrm{C}\right)$ and humidity (40-60\%), and access to food and water ad libitum under a 10:14-h light:dark cycle. Animals were randomly assigned into the following four groups (n=5/group): LV-miR-124; LV-miR-NC; LV-si-STAT3; and LV-si-NC. A total of $5 \times 10^{6}$ stably transfected MDA-MB-468 cells were diluted in $200 \mu \mathrm{l}$ PBS and injected subcutaneously into the right back side of mice. Mice were sacrificed after 3 weeks and tumour sizes were measured. All protocols and procedures were approved by the Animal Care and Use Committee of the Central Hospital of Wuhan.

Statistical analysis. Statistical analysis was conducted using GraphPad Prism version 6.05 (GraphPad Software, Inc., La Jolla, CA, USA). Results are expressed as the mean \pm standard deviation of at least three independent experiments. Comparisons were performed using unpaired Student's t-tests, and one-way and two-way analyses of variance followed by a Student-Newman-Keuls post hoc test. The correlation between the expression of miR-124 and STAT3 was analyzed by Spearman's correlation analysis. $\mathrm{P}<0.05$ was considered to indicate a statistically significant difference.

\section{Results}

miR-124 inhibits the viability and invasion of breast cancer cells in vitro. The downregulation of miR-124 expression in 10 breast cancer tissues and paired normal breast tissues was confirmed by RT-qPCR (Fig. 1A). To evaluate the effects of miR-124 expression on breast cancer cells, MDA-MB-468 and MDA-MB-231 cell lines were transfected with miR-124 (Fig. 1B), and it was identified that the restoration of miR-124 expression suppressed the viability (Fig. 1C) and negatively affected the invasive capacity of these breast cancer cell lines (Fig. 1D). Conversely, downregulation of expression with inh-124 (Fig. 2A) resulted in an increase in cell viability and invasion (Fig. 2B and C). Therefore, miR-124 expression was downregulated in human breast cancer cells and its upregulation prohibited cancer cell invasion in MDA-MB-468 and MDA-MB-231 cells.

STAT3 may serve as the potential target of miR-124 in breast cancer. Bioinformatic studies suggested that STAT3 mRNA may be a target of miR-124; the potential binding area is presented in Fig. 3A. The software prediction was validated by examining the association between miR-124 and STAT3 in 10 pairs of human breast tissues. As demonstrated in Fig. 3B and C, STAT3 was overexpressed in breast cancer tissues, and its expression exhibited a negative correlation with miR-124 expression $\left(\mathrm{r}^{2}=0.37 ; \mathrm{P}=0.01\right)$. Therefore, STAT3 may serve as a potential target of miR-124 in breast cancer cell lines; however, additional in vitro studies are warranted to confirm this hypothesis.

miR-124 negatively regulates the expression of STAT3 via direct interaction in breast cancer. RT-qPCR and western blot analyses were performed to demonstrate 

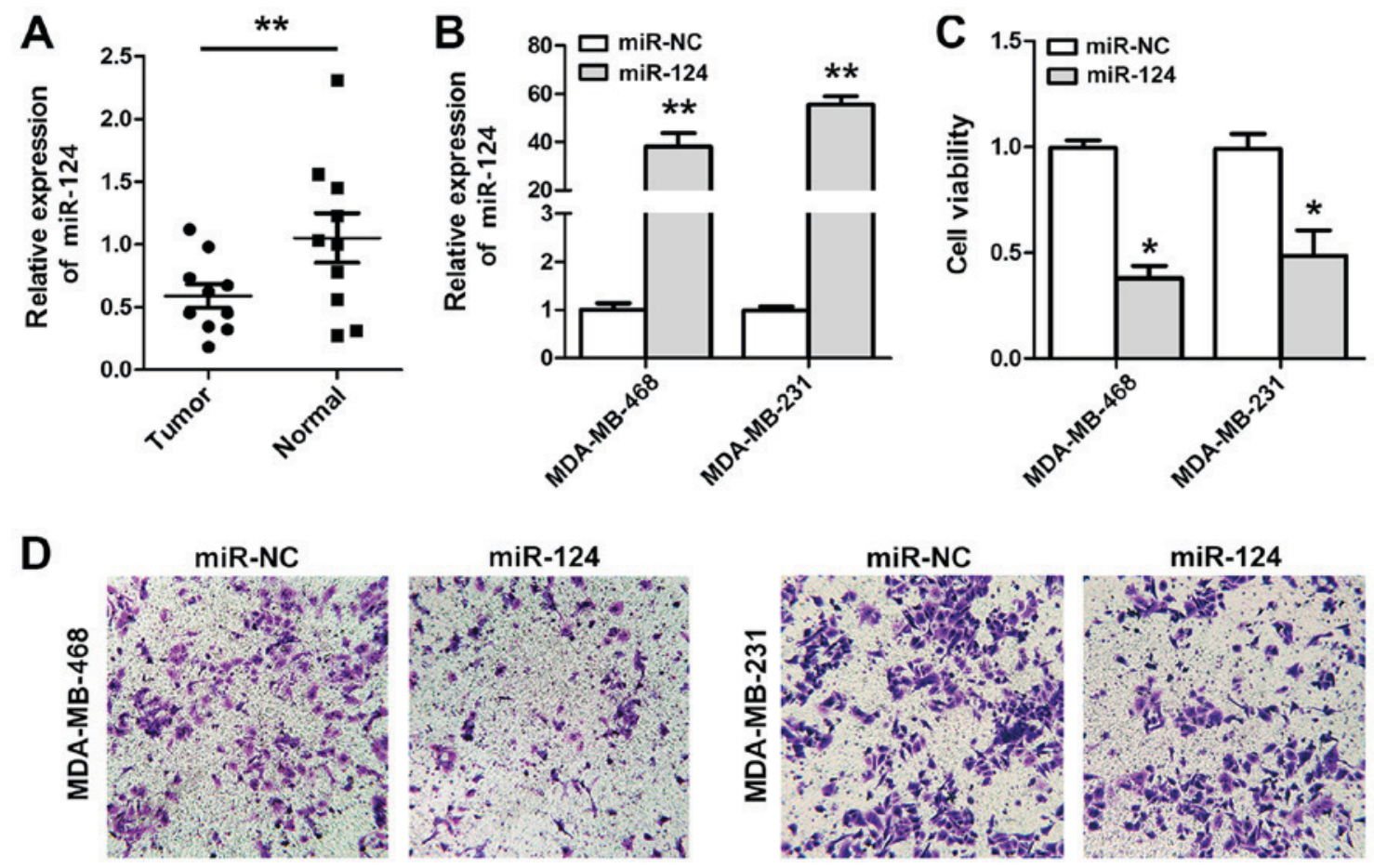

Figure 1. miR-124 is downregulated in breast cancer and suppresses cell viability and invasion in vitro. (A) RT-qPCR was performed to evaluate the expression levels of miR-124 in 20 human breast tissue samples. (B) The efficiency of manual upregulation of miR-124 was determined by RT-qPCR in MDA-MB-468 and MDA-MB-231 cells. (C) Cells were transfected with miR-124 and their viability was measured by MTT assay $48 \mathrm{~h}$ later (D) miR-124 overexpression lead to compromised invasive capacity of breast cancer cells. All data are presented as mean \pm standard deviation of at least three independent experiments. ${ }^{*} \mathrm{P}<0.05$ and ${ }^{* *} \mathrm{P}<0.01$ vs. control. miR-124, microRNA-124; NC, negative control; RT-qPCR, reverse transcription quantitative polymerase chain reaction.
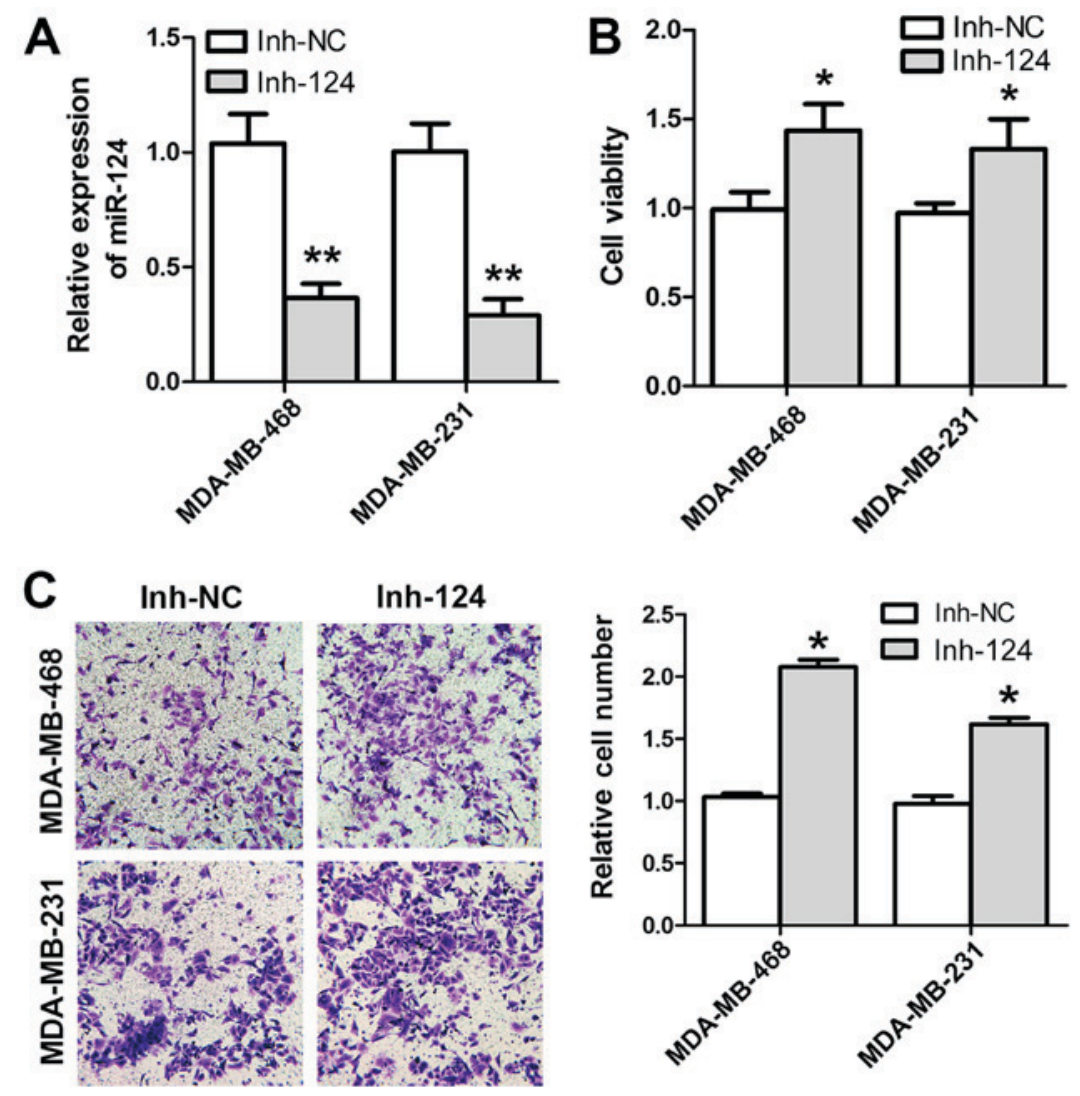

Figure 2. Inhibition of miR-124 promotes cell viability and cell invasion in breast cancer cell lines. (A) Reverse transcription quantitative polymerase chain reaction was performed to evaluate the transfection efficiency of inh-124 (B) Cell viability was measured by MTT assay 2 days post-transfection. (C) Suppressed inh-124 led to enhanced cell invasion in breast cancer cell lines. All data are presented as mean \pm standard deviation of at least three independent experiments. ${ }^{*} \mathrm{P}<0.05$ and ${ }^{* *} \mathrm{P}<0.01$ vs. control. miR-124, microRNA-124; inh, inhibitor; NC, negative control. 
that STAT3 expression was highly downregulated in miR-124-transfected cells. By contrast, inh-124 treatment upregulated STAT3 expression (Fig. 4A and B). Therefore, we hypothesised that miR-124 inhibited cell invasion by regulating the expression of the STAT3 oncogene in breast cancer cells. A dual-luciferase assay was performed to determine whether miR-124 directly interacted with STAT3 mRNA. Dual-luciferase vectors with the STAT3 3'-UTR sequence containing the potential miR-124 response element (WT) or a MUT sequence were co-transfected with miR-124 into MDA-MB-468 cells. miR-124 significantly decreased the firefly luciferase activity of the WT reporter but not the MUT reporter (Fig. 4C). These data indicated that miR-124 may downregulate the expression of STAT3 by binding to its 3'-UTR sequence and modulating the malignant behaviour of breast cancer cells.

Restoration of STAT3 expression rescues the miR-124-induced suppression of MDA-MB-468 cell invasion. Stable expression of miR-124 via lentiviral infection (LV-miR-124) in MDA-MB-468 cells (Fig. 5A) resulted in the downregulation of STAT3 expression. STAT3 levels were restored and cell viability and invasion activities were measured (Fig. 5B). The results indicated that the suppressive effects of LV-miR-124 on cell viability and invasion were partially abrogated following STAT3 overexpression (Fig. 5C and D).

Forced expression of miR-124 and downregulated STAT3 expression abrogates tumour formation in vivo. To determine the functional roles of miR-124 and STAT3 on tumour proliferation, MDA-MB-468 cells were stably transfected with LV-miR-124 and LV-si-STAT3 (Fig. 6). LV-si-STAT3 suppressed the expression of STAT3 and decreased the growth rate of MDA-MB-468 cells (Fig. 7A). A similar result was observed in LV-miR-124-transfected cells. MDA-MB-468 cells stably transfected with LV-miR-124 or LV-si-STAT3 wee subcutaneously injected into the right flanks of mice. Mice were sacrificed 3 weeks later and images of representative tumours from each group were captured (Fig. 7B). The sizes of tumours from mice in the LV-miR-124 and LV-si-STAT3 groups were notably decreased compared with mice from the control group. These results suggested that the overexpression of miR-124 may suppress the proliferation of MDA-MB-468 cells in vivo via the suppression of STAT3 expression.

\section{Discussion}

Cancer is a genetic disease that harbours various gene alterations and mutations (25). Cancer cells exhibit replicative immortality and activated invasion (26). STAT3, a member of the STAT protein family, is activated by Janus kinases in response to cytokines and growth factors. As a result, the activated STAT3 molecule translocates to the cell nucleus and functions as a transcriptional activator to modulate cell growth, apoptosis and invasion $(27,28)$. Constitutive activation of STAT3 has been suggested to contribute to the carcinogenesis of human breast cancer and targeting STAT3 may serve as an effective strategy to suppress the growth and invasion of breast cancer cells (29-31). Liao et al (18) identified that the downregulation of STAT3 expression mediated by miR-17-5p resulted in an increase in chemotherapy-induced apoptosis of breast cancer cells. Furthermore, miR520c-induced degradation of STAT3 resulted in the suppression of EMT progression in breast cancer cells (19). For these reasons, the STAT3 gene appears to be an attractive candidate for mediating the effects of miR-124 in breast cancer.

Regulation of gene expression includes a wide range of mechanisms, including DNA-RNA transcription to post-translational modifications of the proteins (32-33). Previous evidence has suggested that miRNAs bind to the complementary sequences within the 3'-UTR of specific target mRNAs to suppress the post-translational expression of genes either by mRNA degradation or translational inhibition $(6,7)$. miRNAs have been associated with cancer through their regulatory effects on tumour-relevant genes and effects on numerous cancer-associated cellular behaviours, including cell invasion, proliferation and chemoresistance $(6,7)$. Therefore, the evaluation of the regulatory effects of miRNAs is valuable for the understanding of the underlying mechanism of STAT3 overexpression in breast cancer tissues.

Prediction of the association between novel miRNAs and diseases is of clinical significance. Certain new valuable computational tools for miRNA-disease association prediction are available, including Laplacian Regularized Sparse Subspace Learning for miRNA-disease association prediction (34), Matrix decomposition and Heterogeneous Graph Interference for miRNA-disease association prediction (35) and Bipartite Network Projection for miRNA-disease association prediction (36). These software programmes offer the advantages of high levels of efficiency and accuracy. A total of $>95 \%$ of the top 50 predictions associated with various tumors are validated by conventional open databases (35). In the present study, the bioinformatic analysis predicted a physical interaction between miR-124 and the 3'-UTR of STAT3 mRNA, suggesting that STAT3 may be a target candidate of miR-124. miR-124 expression is downregulated in numerous cancer tissues, including breast cancer $(14,15)$, and ectopic expression of miR-124 resulted in the attenuation of cell growth and invasion in the present study. Together these data support the tumour-suppressive role of miR-124 in human breast cancer.

In the present study, miR-124 was transfected into the breast cancer MDA-MB-468 and MDA-MB-231 cell lines, and its effects on cancer phenotypes were evaluated. Expression of miR-124 significantly suppressed the viability, proliferation and invasion of breast cancer cells through the downregulation of STAT3 expression. Similar results were also observed in the in vivo studies, wherein the overexpression of miR-124 resulted in a decrease in tumour sizes. These data suggest that the loss of miR-124 expression results in an increase in STAT3 expression, leading to promotion of cell viability, growth rate and enhanced invasive capacity. Therefore, we hypothesise that the manual restoration of miR-124 expression may be an effective therapeutic strategy for the treatment of patients with breast cancer. In comparison with normal breast tissues, breast cancer tissues exhibited a significantly decreased expression of miR-124. In addition, the data obtained from the in vitro and in vivo studies demonstrated the antitumour function of miR-124 was mediated through the downregulation of the expression 

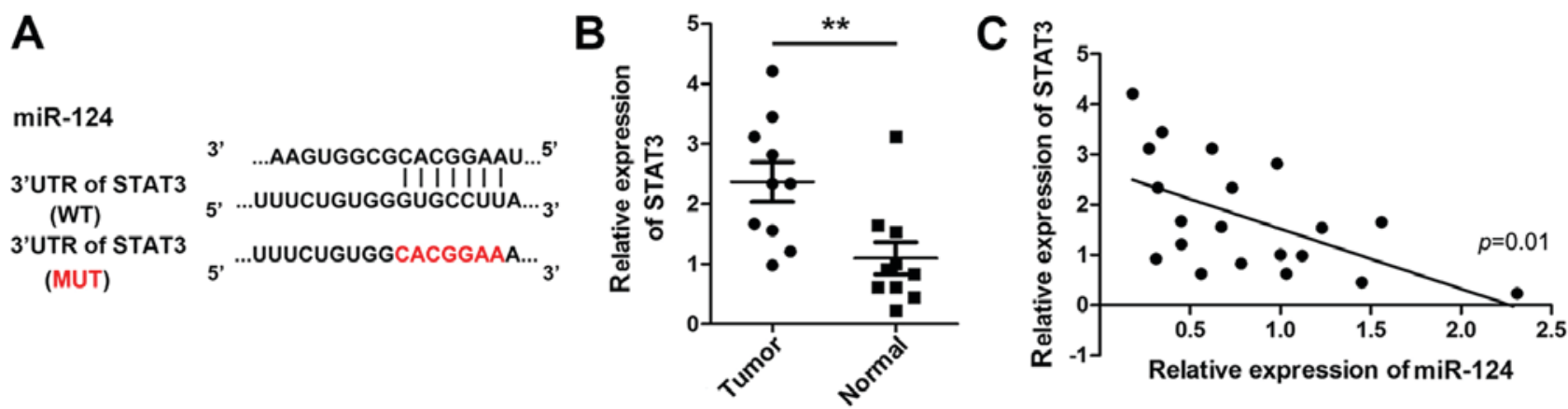

Figure 3. STAT3 may be a downstream of miR-124 in breast cancer. (A) The potential miR-124 binding area in the WT 3'UTR of STAT3 mRNA, the corresponding MUT sequence is in red. (B) Reverse transcription quantitative polymerase chain reaction was performed to measure the expression of miR-124 in human breast tissues. (C) The correlation between expression of miR-124 and STAT3 in 20 human breast tissue samples. "P $<0.01$ vs. control. STAT3, signal transducer and activator of transcription 3; UTR, untranslated region; miR-124, microRNA-124; WT, wide type; MUT, mutated.
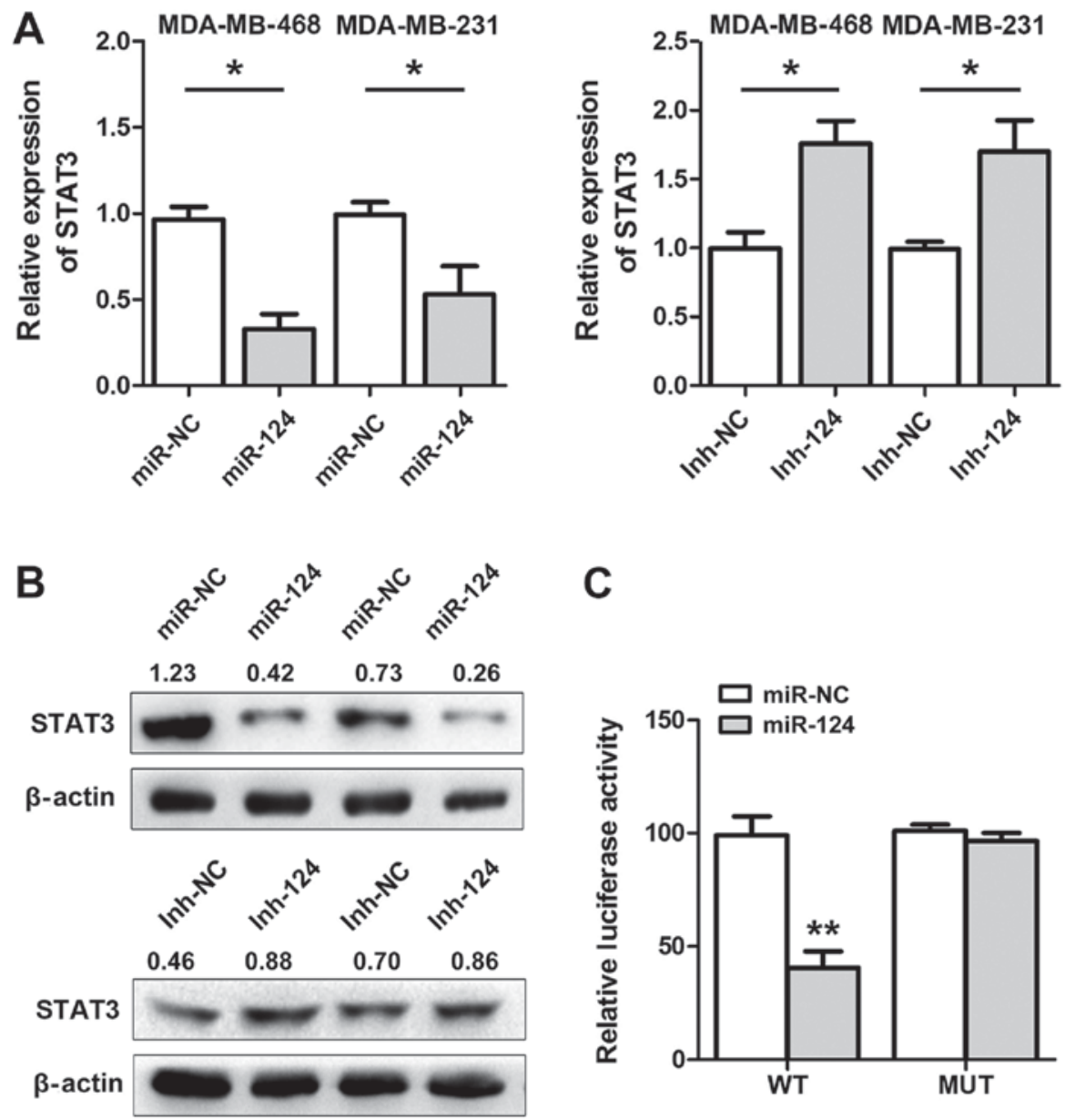

Figure 4. miR-124 negatively regulates the expression of STAT3 by direct interaction in breast cancer. (A) The expression of STAT3 mRNA was decreased by overexpression of miR-124 in MDA-MB-468 and MDA-MB-231 cells, while inhibition of miR-124 led to the upregulation of STAT3. (B) The corresponding western blot data of STAT3. Data values represent the relative intensity values. (C) A dual luciferase assay was performed following co-transfection with WT or MUT reporters and miR-124 or miR-NC in MDA-MB-468 cells. miR-124 overexpression lead to a significant decrease in the activity of the WT reporter but not the MUT reporter. Firefly luciferase activity was used to normalize the data. All data are presented as mean \pm standard deviation of at least three independent experiments. ${ }^{*} \mathrm{P}<0.05$ and ${ }^{* *} \mathrm{P}<0.01$ vs. control. miR-124, microRNA-124; STAT3, signal transducer and activator of transcription 3 ; inh, inhibitor; miR-124, microRNA-124; WT, wild type; MUT, mutant; NC, negative control.

of the oncogene STAT3 in breast cancer cell lines, consistent with the prediction of the bioinformatics analysis. The direct interaction between miR-124 and the 3'-UTR of STAT3 in MDA-MB-468 cells was verified using a dual-luciferase assay. The restoration of STAT3 expression upon transfection with the plasmid-STAT3 into the cells stably overexpressing miR-124 abolished the suppressive effects of miR-124 on cell growth and invasion. Taken together, these data indicate that miR-124 suppresses the growth and invasion of breast cancer cells via downregulation of STAT3 expression. 

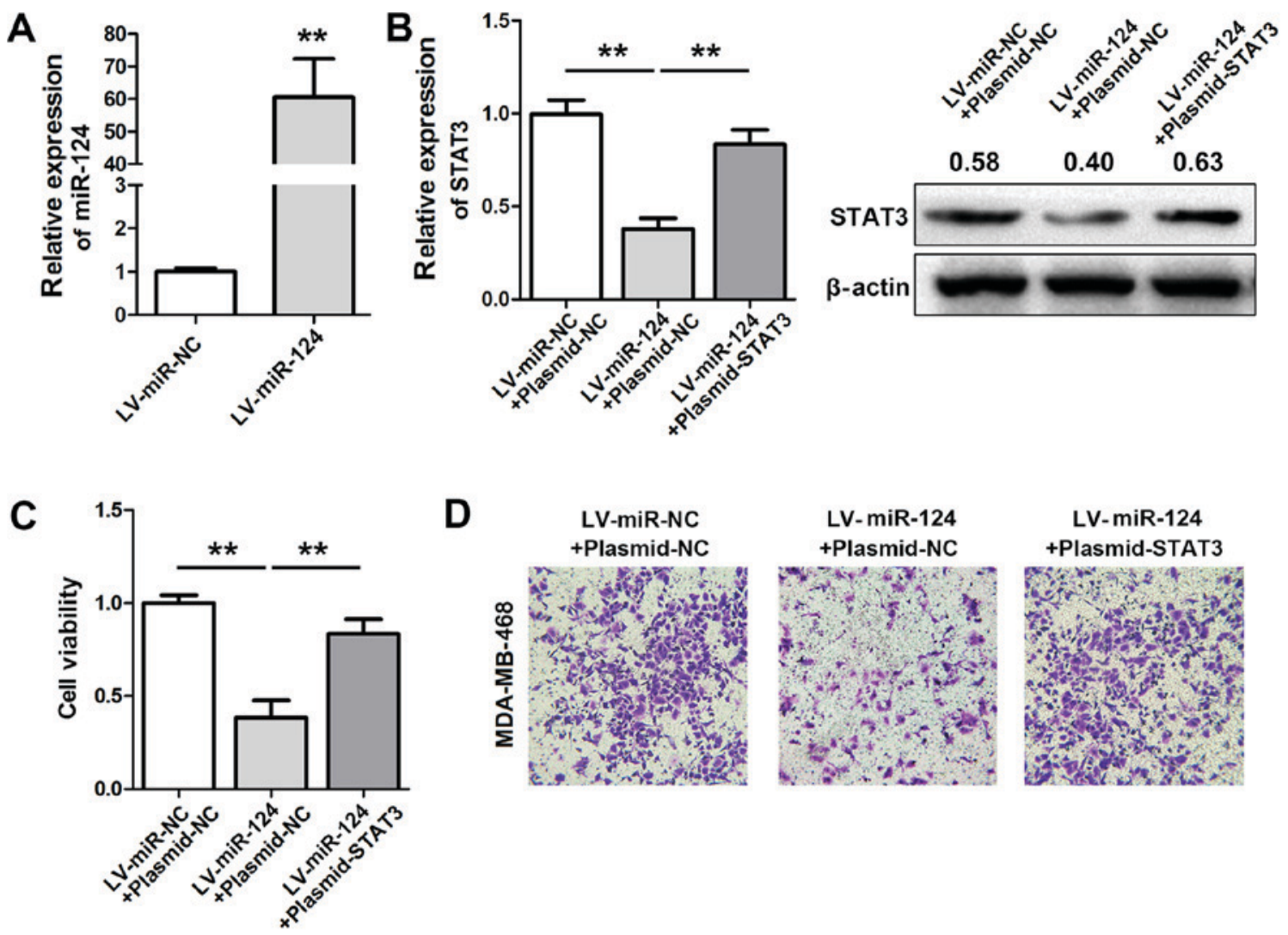

D
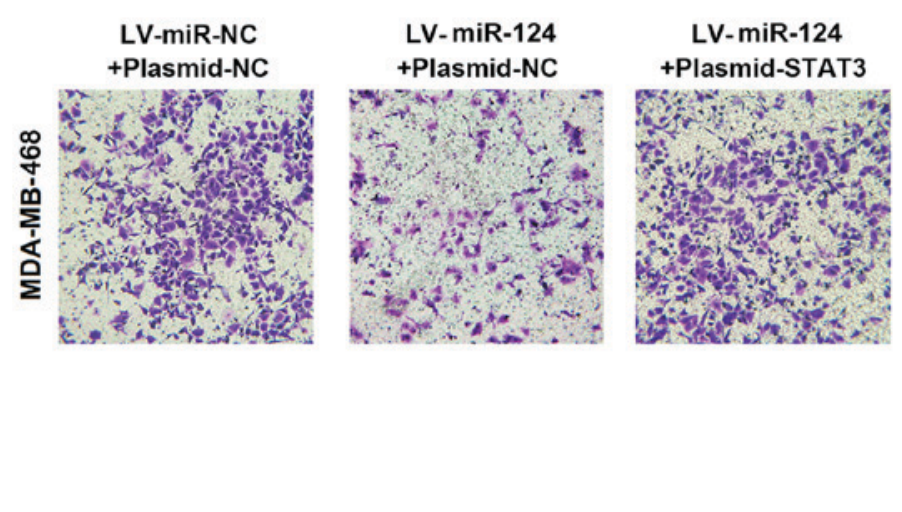

Figure 5. Restoration of STAT3 impairs the prohibitive effects of miR-124 on cell viability and invasion in MDA-MB-468. (A) miR-124 expression levels in MDA-MB-468 cells infected with LV-miR-124. (B) Stable expression of miR-124 suppressed the expression of STAT3. However, STAT3 mRNA and protein levels were restored by transfection with plasmid-STAT3. Data values represent the relative intensity value. (C) STAT3 overexpression reversed the prohibitive cell viability caused by LV-miR-124. (D) A significantly suppressed invasive capacity was detected in MDA-MB-468 cells subsequent to LV-miR-124 transfection. This was partially recovered following plasmid-STAT3 transfection. All data are presented as mean \pm standard deviation of at least three independent experiments. ${ }^{* *} \mathrm{P}<0.01$ vs. control. STAT3, signal transducer and activator of transcription 3; miR-124, microRNA-124; LV-miR-124, miR-124 expression lentivirus; plasmid-STAT3, STAT3 expression plasmid; NC, negative control.
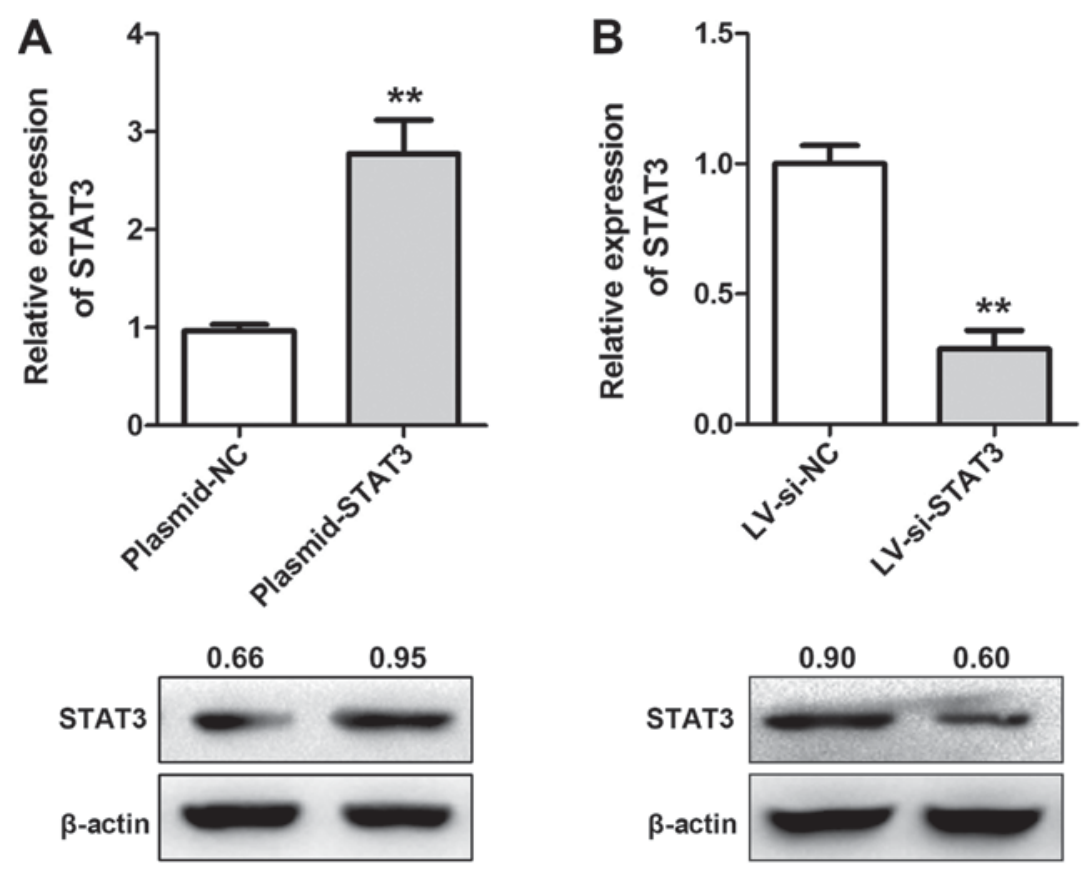

Figure 6. Transfection efficiency of plasmid-STAT3 and LV-si-STAT3 in MDA-MB-468 cells. (A) The transfection efficiency of plasmid-STAT3 was measured using RT-qPCR assays and western blot analysis. (B) RT-qPCR assays and western blot analysis were performed to evaluate the transfection efficiency of LV-si-STAT3. All data are presented as mean \pm standard deviation of at least three independent experiments. Data values included in the western blot gel images represent the relative intensity value of the bands. ${ }^{* *} \mathrm{P}<0.01$ vs. control. STAT3, signal transducer and activator of transcription 3 ; siRNA, small interfering RNA; LV-si-STAT3, si-STAT3 lentivirus; RT-qPCR, reverse transcription quantitative polymerase chain reaction. 


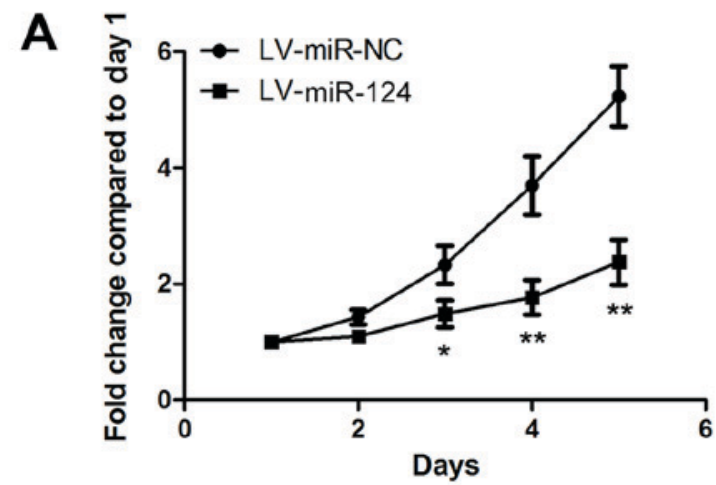

B
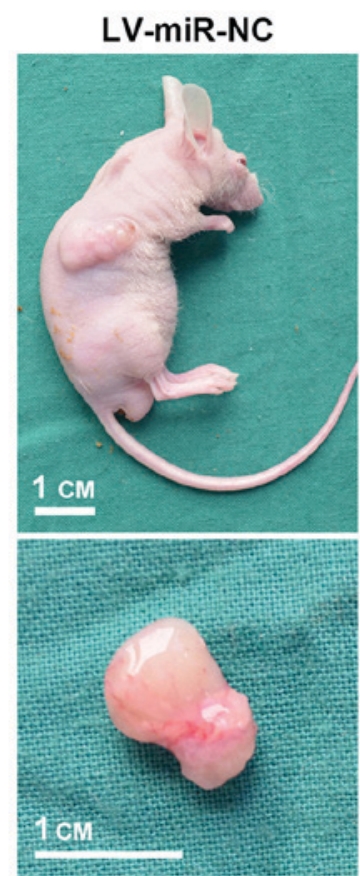

LV-miR-124
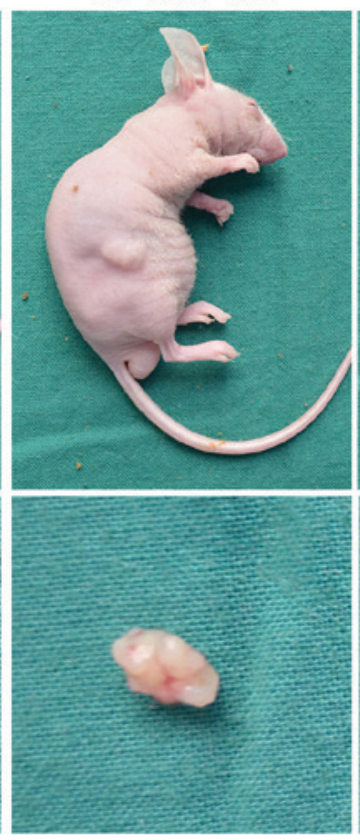

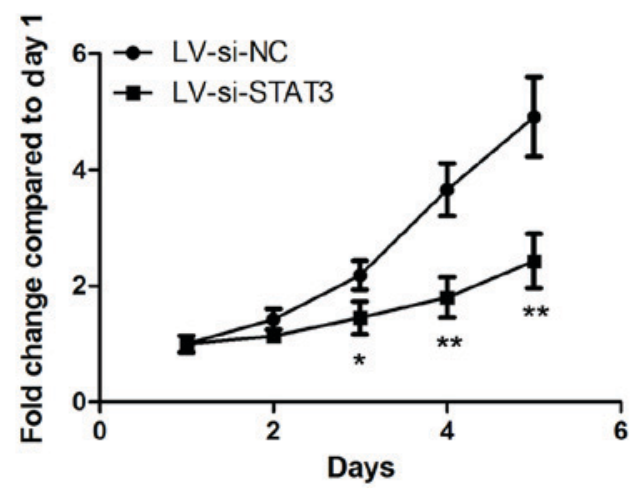

LV-si-NC
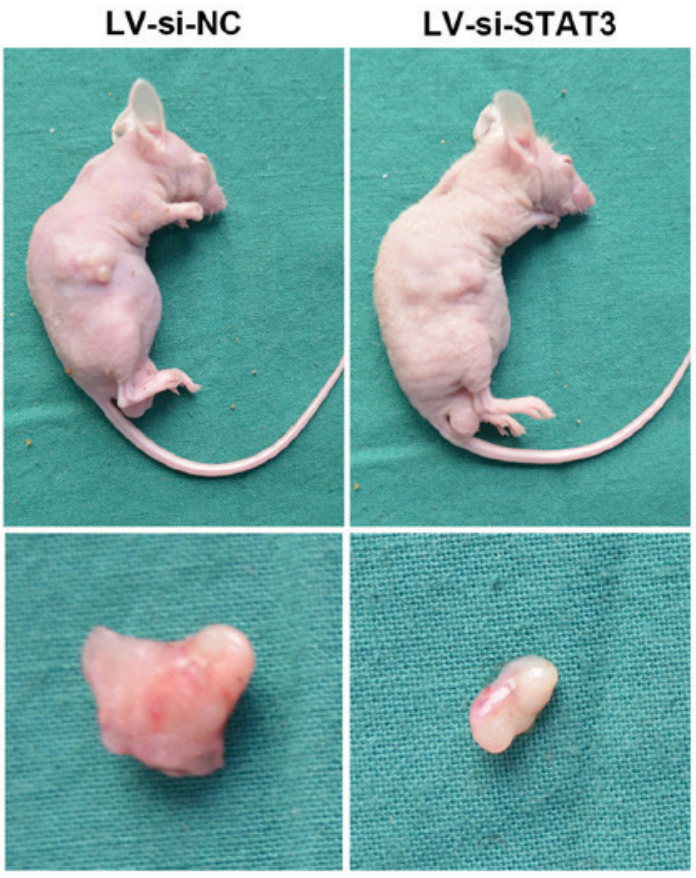

Figure 7. Overexpression of miR-124 and downregulation of STAT3 lead to smaller tumor sizes in vivo. (A) The proliferation curves of MDA-MB-468 cells following stable transfection of miR-124 or LV-si-STAT3 were obtained after 5 days of observation. (B) The representative nude mice and corresponding tumors for the four groups at 3 weeks post-inoculation. ${ }^{*} \mathrm{P}<0.05$ and ${ }^{* *} \mathrm{P}<0.01$ vs. control. miR-124, microRNA-124; STAT3, signal transducer and activator of transcription 3; siRNA, small interfering RNA; LV-si-STAT3, si-STAT3 lentivirus.

\section{Acknowledgements}

Not applicable.

\section{Funding}

The present study was funded by the National Science Foundation Committee of China (grant nos. 81600366 and 81702332).

\section{Availability of data and materials}

The data used and/or analyzed during the current study are available from the corresponding author on reasonable request.

\section{Authors' contributions}

YL made substantial contributions towards the design of the study and analyzed the data. PS, CC and ZW performed the in vitro and in vivo experiments. XL and ZL collected the clinical and histologic data. PS drafted the manuscript.

\section{Ethics approval and consent to participate}

The study protocol followed the Declaration of Helsinki, and all patients provided written informed consent. All protocols and procedures were approved by the Ethics Committee of the Central Hospital of Wuhan and the Animal Care and Use Committee of the Central Hospital of Wuhan.

\section{Patient consent for publication}

All patients provided written informed consent.

\section{Competing interests}

The authors declare that they have no competing interests.

\section{References}

1. DeSantis C, Ma J, Bryan L and Jemal A: Breast cancer statistics, 2013. CA Cancer J Clin 64: 52-62, 2014. 
2. Li Y, Cai B, Shen L, Dong Y, Lu Q, Sun S, Liu S, Ma S, Ma PX and Chen J: MiRNA-29b suppresses tumor growth through simultaneously inhibiting angiogenesis and tumorigenesis by targeting Akt3. Cancer Lett 397: 111-119, 2017.

3. Sun G, Sun L, Liu Y, Xing H and Wang K: Her-2 expression regulated by downregulation of miR-9 and which affects chemotherapeutic effect in breast cancer. Cancer Gene Ther 24: 194-202, 2017.

4. Liu Y, Li X, Zhu S, Zhang JG, Yang M, Qin Q, Deng SC, Wang B, Tian K, Liu L, et al: Ectopic expression of miR-494 inhibited the proliferation, invasion and chemoresistance of pancreatic cancer by regulating SIRT1 and c-Myc. Gene Ther 22: 729-738, 2015.

5. Li X, Deng SJ, Zhu S, Jin Y, Cui SP, Chen JY, Xiang C, Li QY, He C, Zhao SF, et al: Hypoxia-induced lncRNA-NUTF2P3-001 contributes to tumorigenesis of pancreatic cancer by derepressing the miR-3923/KRAS pathway. Oncotarget 7: 6000-6014, 2016.

6. Bartel DP: MicroRNAs: Genomics, biogenesis, mechanism, and function. Cell 116: 281-297, 2004.

7. Winter J, Jung S, Keller S, Gregory RI and Diederichs S: Many roads to maturity: microRNA biogenesis pathways and their regulation. Nat Cell Biol 11: 228-234, 2009.

8. Wang B, Zou A, Ma L, Chen X, Wang L, Zeng X and Tan T: miR-455 inhibits breast cancer cell proliferation through targeting CDK14. Eur J Pharmacol 807: 138-143, 2017.

9. Li P, Xu T, Zhou X, Liao L, Pang G, Luo W, Han L, Zhang J, Luo X, Xie X and Zhu K: Downregulation of miRNA-141 in breast cancer cells is associated with cell migration and invasion: Involvement of ANP32E targeting. Cancer Med 6: 662-672, 2017.

10. Zhao M, Ang L, Huang J and Wang J: MicroRNAs regulate the epithelial-mesenchymal transition and influence breast cancer invasion and metastasis. Tumour Biol 39: 1010428317691682, 2017.

11. Chen Z, Liu S, Tian L, Wu M, Ai F, Tang W, Zhao L, Ding J, Zhang L and Tang A: miR-124 and miR-506 inhibit colorectal cancer progression by targeting DNMT3B and DNMT1. Oncotarget 6: 38139-38150, 2015.

12. Sun Y, Ai X, Shen S and Lu S: NF- $\kappa B-m e d i a t e d ~ m i R-124$ suppresses metastasis of non-small-cell lung cancer by targeting MYO10. Oncotarget 6: 8244-8254, 2015.

13. Peng XH, Huang HR, Lu J, Liu X, Zhao FP, Zhang B, Lin SX, Wang L, Chen $\mathrm{HH}, \mathrm{Xu} \mathrm{X}$, et al: MiR-124 suppresses tumor growth and metastasis by targeting Foxq1 in nasopharyngeal carcinoma. Mol Cancer 13: 186, 2014.

14. Cai WL, Huang WD, Li B, Chen TR, Li ZX, Zhao CL, Li HY, Wu YM, Yan WJ and Xiao JR: microRNA-124 inhibits bone metastasis of breast cancer by repressing Interleukin-11. Mol Cancer 17: 9, 2018.

15. Du S, Li H, Sun X, Li D, Yang Y, Tao Z, Li Q and Liu K: MicroRNA-124 inhibits cell proliferation and migration by regulating SNAI2 in breast cancer. Oncol Rep 36: 3259-3266, 2016.

16. Wang Y, Chen L, Wu Z, Wang M, Jin F, Wang N, Hu X, Liu Z, Zhang CY,Zen K, et al: miR-124-3p functions as a tumor suppressor in breast cancer by targeting CBL. BMC Cancer 16: 826, 2016.

17. Feng T, Shao F, Wu Q, Zhang X, Xu D, Qian K, Xie Y, Wang S, $\mathrm{Xu}$ N, Wang Y and Qi C: miR-124 downregulation leads to breast cancer progression via LncRNA-MALAT1 regulation and CDK4/E2F1 signal activation. Oncotarget 7: 16205-16216, 2016.

18. Liao XH, Xiang Y, Yu CX, Li JP, Li H, Nie Q, Hu P, Zhou J and Zhang TC: STAT3 is required for MiR-17-5p-mediated sensitization to chemotherapy-induced apoptosis in breast cancer cells Oncotarget 8: 15763-15774, 2016.

19. Wang N, Wei L, Huang Y, Wu Y, Su M, Pang X, Wang N, Ji F, Zhong C, Chen T and Li B: miR520c blocks EMT progression of human breast cancer cells by repressing STAT3. Oncol Rep 37: $1537-1544,2017$
20. Liu F, Zhang H and Song H: Upregulation of MEK5 by Stat3 promotes breast cancer cell invasion and metastasis. Oncol Rep 37: 83-90, 2017.

21. McDaniel JM, Varley KE, Gertz J, Savic DS, Roberts BS, Bailey SK, Shevde LA, Ramaker RC, Lasseigne BN, Kirby MK, et al: Genomic regulation of invasion by STAT3 in triple negative breast cancer. Oncotarget 8: 8226-8238, 2017.

22. Xiang Y, Liao XH, Yu CX, Yao A, Qin H, Li JP, Hu P, Li H, Guo W, Gu CJ and Zhang TC: MiR-93-5p inhibits the EMT of breast cancer cells via targeting MKL-1 and STAT3. Exp Cell Res 357: 135-144, 2017

23. Livak KJ and Schmittgen TD: Analysis of relative gene expression data using real-time quantitative PCR and the 2(-Delta Delta C(T)) method. Methods 25: 402-408, 2001

24. Kertesz M, Iovino N, Unnerstall U, Gaul U and Segal E: The role of site accessibility in microRNA target recognition. Nat Genet 39: 1278-1284, 2007.

25. Watson IR, Takahashi K, Futreal PA and Chin L: Emerging patterns of somatic mutations in cancer. Nat Rev Genet 14: 703-718, 2013.

26. Hanahan D and Weinberg RA: Hallmarks of cancer: The next generation. Cell 144: 646-674, 2011.

27. Ilamathi M, Prabu PC, Ayyappa KA and Sivaramakrishnan V: Artesunate obliterates experimental hepatocellular carcinoma in rats through suppression of IL-6-JAK-STAT signalling. Biomed Pharmacother 82: 72-79, 2016.

28. Siveen KS, Sikka S, Surana R, Dai X, Zhang J, Kumar AP, Tan BK, Sethi G and Bishayee A: Targeting the STAT3 signaling pathway in cancer: Role of synthetic and natural inhibitors. Biochim Biophys Acta 1845: 136-154, 2014.

29. Tkach M, Rosemblit C, Rivas MA, Proietti CJ, Díaz Flaqué MC, Mercogliano MF, Beguelin W, Maronna E, Guzmán P, Gercovich FG, et al: p42/p44 MAPK-mediated Stat3Ser727 phosphorylation is required for progestin-induced full activation of Stat 3 and breast cancer growth. Endocr Relat Cancer 20 197-212, 2013.

30. Banerjee $\mathrm{K}$ and Resat $\mathrm{H}$ : Constitutive activation of STAT3 in breast cancer cells: A review. Int J Cancer 138: 2570-2578, 2016.

31. Thakur R, Trivedi R, Rastogi N, Singh M and Mishra DP: Inhibition of STAT3, FAK and Src mediated signaling reduces cancer stem cell load, tumorigenic potential and metastasis in breast cancer. Sci Rep 5: 10194, 2015.

32. Baek D, Villén J, Shin C, Camargo FD, Gygi SP and Bartel DP: The impact of microRNAs on protein output. Nature 455: 64-71, 2008.

33. Bird A: DNA methylation patterns and epigenetic memory. Genes Dev 16: 6-21, 2002.

34. Chen X and Huang L: LRSSLMDA: Laplacian regularized sparse subspace learning for MiRNA-disease association prediction. PLoS Comput Biol 13: e1005912, 2017.

35. Chen X, Yin J, Qu J and Huang L: MDHGI: Matrix decomposition and heterogeneous graph inference for miRNA-disease association prediction. PLoS Comput Biol 14: e1006418, 2018.

36. Chen X, Xie D, Wang L, Zhao Q, You ZH and Liu H: BNPMDA: Bipartite network projection for MiRNA-disease association prediction. Bioinformatics 34: 3178-3186, 2018.

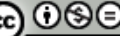

This work is licensed under a Creative Commons Attribution-NonCommercial-NoDerivatives 4.0 International (CC BY-NC-ND 4.0) License. 